Pacific Journal of Mathematics

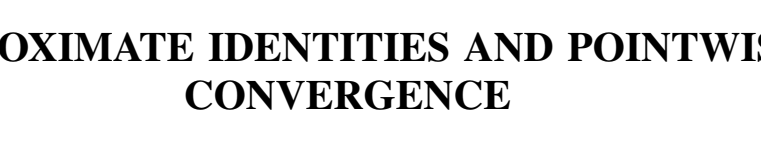




\title{
APPROXIMATE IDENTITIES AND POINTWISE CONVERGENCE
}

\author{
H. S. BEAR
}

\begin{abstract}
We give two additional condit!ons on an approximate identity (or positive kernel) $\left\{K_{\alpha}\right\}$ which insure that $f^{*} K_{\alpha} \rightarrow f$ a.e. if $f \in L^{1}$ on the line or circle. Where the convolution defines a function on the disc or a half-plane, as for the Poisson kernels or heat kernels, then the theorem gives automatically the paths toward a boundary point along which pointwise convergence occurs.
\end{abstract}

1. Introduction. An approximate identity on the line or the circle is a family of bounded nonnegative $L^{1}$ functions $\left\{K_{\alpha}\right\}$ such that $\int K_{\alpha}=1$ and $\lim _{\alpha} \int_{I} K_{\alpha}=1$ for all intervals $I=\{x:|x| \leqq \delta\}, \delta>0$. The convolutions $f_{\alpha}=f * K_{\alpha}$ of a given function $f$ with the members of an approximate identity provide approximations which converge to $f$ in various ways depending on $f$. For a finite interval, for example, $f_{\alpha} \rightarrow f$ uniformly if $f$ is continuous; $f_{\alpha} \rightarrow f$ in $L^{p}$ if $f \in L^{p}(1 \leqq p<\infty) ; f_{\alpha} \rightarrow f w^{*}$ if $f \in L_{\infty}$ [3, p. 22].

For specific approximate identities (Poisson kernels, heat kernels, the Fejér kernel) one also has $f_{\alpha} \rightarrow f$ pointwise almost everywhere. The proofs of these theorems use additional properties of the several kernels beyond the very general conditions for an approximate identity.

To illustrate, the Poisson kernel for the disc is

$$
P_{r}(\theta)=\frac{1}{2 \pi} \frac{1-r^{2}}{1+r^{2}-2 r \cos \theta} . \quad(0 \leqq r<1 ;-\pi<\theta \leqq \pi) .
$$

If $f \in L^{1}(-\pi, \pi)$, let $f(r, \theta)=f_{r}(\theta)=\left(f * P_{r}\right)(\theta)$. Then $f(r, \theta)$ is harmonic in $|z|<1$, and $f(r, \theta) \rightarrow f(\theta)$ a.e. as $r \rightarrow 1$. In fact, one actually has the following classical theorem on nontangential approach: $f(r, \theta) \rightarrow f(\theta)$ a.e. as $(r, \theta) \rightarrow(1, \theta)$ along any nontangential path.

Our purpose here is to prove a theorem of this form: If $f \in L^{1}$ and $f_{\alpha}=f * K_{\alpha}$, then $f_{\alpha} \rightarrow f$ a.e. The hypothesis is that $\left\{K_{\alpha}\right\}$ be an approximate identity (on the circle or line) with two additional assumptions. The first of these is simply a smoothness assumption: $K_{\alpha}^{\prime}$ is continuous, and each $K_{\alpha}$ has a unique maximum, and decreases monotonically away from this maximum in both directions. The second extra assumption (condition (e)) limits the distance from the origin at which $K_{\alpha}$ can have its maximum.

In the applications, condition (e) determines which translates of 
a given approximate identity can be added to the family so the result will still be an approximate identity. For the Poisson kernels, condition (e) is equivalent to restricting approach to a boundary point to paths within a Stolz angle. Hence condition (e) is in some sense "best possible."

Applied to the heat kernels our theorem gives apparently new results on the kind of approach toward a boundary point for which heat convolutions converge to the boundary function a.e.

For the Fejér kernel, "approach to a boundary point" is not a relevant idea. However, we do obtain information about how the modulus of continuity of the Cesáro sums of an $L^{1}$ function depend on $n$. The same sort of inference can be made for the other kernels, and is basically what is involved in the results on "nonperpendicular" approach.

There is nothing novel in the proof of the theorem. What is new is the isolation of the simple conditions which make all the standard proofs work, and the fact that translates of an approximate identity again form an approximate identity when suitably indexed. It is this last fact which gives the paths toward boundary points along which pointwise convergence takes place.

2. Proof of the theorem. Let $\left\{K_{\alpha}\right\}$ be a net of nonnegative real functions on $X=(-\infty, \infty)$, or on $X=(-\pi, \pi]$ (the circle). The index $\alpha$ is an element of a set $D$ with a transitive partial ordering $\succ$. In addition we assume that for every $\alpha_{1}, \alpha_{2} \in D$, there is $\alpha_{3} \in D$ so that $\alpha_{3}>\alpha_{1}$ and $\alpha_{3}>\alpha_{2}$. Thus $(D,>)$ is a directed set. We write $\alpha \rightarrow \infty$ to indicate limits as $\alpha$ runs over $D$; e.g., $K_{\alpha}\left(x_{\jmath}\right) \rightarrow 0$ as $\alpha \rightarrow \infty$.

The net $\left\{K_{\alpha}\right\}$ is an approximate identity if (a), (b), (c) below are satisfied, and we will call $\left\{K_{\alpha}\right\}$ a smooth approximate identity if in addition (d) and (e) are satisfied.

(a) $K_{\alpha} \in L^{1}$ and $K_{\alpha} \geqq 0$ for all $\alpha$.

(b) $\int K_{\alpha}=1$ for all $\alpha$.

(c) $\int_{|x| \leq \delta} K_{\alpha} \rightarrow 1$ as $\alpha \rightarrow \infty$, for all $\delta>0$.

(d) $K_{\alpha}^{\prime}$ is continuous on $X$. $K_{\alpha}$ increases to a unique maximum at $x_{\alpha}$, and decreases for $x \geqq x_{\alpha}$. For the circle, $K_{a}$ increases from some minimum value along the two complementary arcs to a unique maximum at $x_{\alpha}$.

(e) For some constant $A,\left|x_{\alpha}\right| K_{\alpha}\left(x_{\alpha}\right) \leqq A$ for all $\alpha$.

We will prove the following theorem.

THEOREM 1. If $f \in L^{1}$ on $X$ and $\left\{K_{\alpha}\right\}$ is a smooth approximate identity, then $f * K_{\alpha}(x) \rightarrow f(x)$ a.e. as $\alpha \rightarrow \infty$. 
The proof depends on a number of simple lemmas. Throughout this section we assume that $\left\{K_{\alpha}\right\}$ is a smooth approximate identity on $X$, where $X$ is either the line or the circle. The notation will be for the case $X=(-\infty, \infty)$.

LeMma 1. $\int_{|x| \geq o} K_{\alpha} \rightarrow 0$ as $\alpha \rightarrow \infty$ for all $\delta>0$.

Proof. This is immediate from (b) and (c).

Lemma 2. $x_{\alpha} \rightarrow 0$ as $\alpha \rightarrow \infty$.

Proof. By (c), $\max K_{\alpha}=K_{\alpha}\left(x_{\alpha}\right) \rightarrow \infty$ as $\alpha \rightarrow \infty$. Hence $x_{\alpha} \rightarrow 0$ by (e).

Lemma 3. $\sup \left\{K_{\alpha}(s):|s| \geqq \delta\right\} \rightarrow 0$ as $\alpha \rightarrow \infty$, for all $\delta>0$. In particular, $K_{\alpha}\left(s_{0}\right) \rightarrow 0$ as $\alpha \rightarrow \infty$ for all $s_{0} \neq 0$.

Proof. Fix $\delta>0$. Then $\left|x_{\alpha}\right|<\delta$ for all sufficiently "large" $\alpha$, and $\sup \left\{K_{\alpha}(s):|s| \geqq \delta\right\}$ is either $K_{\alpha}(\delta)$ or $K_{\alpha}(-\delta)$. Suppose the lemma is false, and to be specific that there are arbitrarily large values of $\alpha$ for which $K_{\alpha}(\delta) \geqq p>0$. Then there are arbitrarily large values of $\alpha$ such that $\left|x_{\alpha}\right| \leqq \delta / 2$ and $K_{\alpha}(\delta) \geqq p$ by Lemma 2. For all such $\alpha, \int_{|x| \geq \delta / 2} K_{\alpha} \geqq 1 / 2 \delta p$, which contradicts Lemma 1 .

The next lemma is relevant only if $X=(-\infty, \infty)$.

Lemma 4. $\lim _{|s| \rightarrow \infty} K_{\alpha}(s)=0$ for all $\alpha$.

Proof. This follows from (d) and the fact that $K_{\alpha} \in L^{1}$.

LemMA 5. For all $\delta>0, \int_{|x| \geqq \delta} K_{\alpha}^{\prime} \rightarrow 0$ as $\alpha \rightarrow \infty$.

Proof. We consider the interval $[\delta, \infty)$. The proof for the other cases is similar. For each $\alpha, K_{\alpha}^{\prime} \in L^{1}$ by (d) and Lemma 3, and

$$
\int_{\delta}^{\infty} K_{\alpha}^{\prime}=\lim _{s \rightarrow \infty} K_{\alpha}(s)-K_{\alpha}(\delta)=-K_{\alpha}(\delta)
$$

As $\alpha \rightarrow \infty, K_{\alpha}(\delta) \rightarrow 0$.

The next lemma uses condition (e) in an essential way. Condition (e) determines the paths toward boundary points (i.e., points $x_{0}$ of $X$ ) along which the convolutions $f * K_{\alpha}$ will approach $f\left(x_{0}\right)$ a.e. 
Lemma 6. There is a constant $B$ and $\alpha_{0} \in D$ such that $\int\left|x K_{\alpha}^{\prime}(x)\right| d x \leqq B$ for all $\alpha>\alpha_{0}$.

Proof. Fix $M>0$, and pick $\alpha_{0}$ so that $\left|x_{\alpha}\right|<M$ for $\alpha \succ \alpha_{0}$. Now we consider only $\alpha \succ \alpha_{0}$, and show that $\int_{-M}^{M}\left|x K_{\alpha}^{\prime}(x)\right| d x \leqq B$, where $B$ is independent of $M$. Fix $\alpha$, and assume $0 \leqq x_{\alpha}<M$; the same sort of argument works if $-M<x_{\alpha} \leqq 0$.

$$
\begin{aligned}
\int_{-M}^{M I}\left|x K_{\alpha}^{\prime}(x)\right| d x= & \int_{-M}^{0}-x K^{\prime}(x) d x+\int_{0}^{x_{\alpha}} x K_{\alpha}^{\prime}(x) d x-\int_{x_{\alpha}}^{M} x K^{\prime}(x) d x \\
= & \left.-x K_{\alpha}(x)\right]_{-M}^{0}+\int_{-M}^{0} K_{\alpha}(x) d x \\
& \left.+x K_{\alpha}(x)\right]_{0}^{x_{\alpha}}-\int_{0}^{x_{\alpha}} K_{\alpha}(x) d x \\
& \left.-x K_{\alpha}(x)\right]_{x_{\alpha}}^{M}+\int_{x_{\alpha}}^{M} K_{\alpha}(x) d x \\
\leqq & (-M) K_{\alpha}(-M)+x_{\alpha} K_{\alpha}\left(x_{\alpha}\right)-M K_{\alpha}(M)+x_{\alpha} K_{\alpha}\left(x_{\alpha}\right)+1 \\
\leqq & 2 x_{\alpha} K_{\alpha}\left(x_{\alpha}\right)+1 \leqq 2 A+1 \equiv B .
\end{aligned}
$$

LEMMA 7. If $f \in L^{1}$, then for almost all $x$,

$$
\lim _{s \rightarrow x} \frac{1}{s-x} \int_{x}^{s}(f(u)-f(x)) d u=0 .
$$

Proof. If $F(x)=\int_{0}^{x} f(u) d u$, then $F^{\prime}(x)=f(x)$ a.e.; i.e., $(F(s)-F(x)) /$ $(s-x) \rightarrow f(x)$ a.e., which is the same as (1).

Corollary. The limit (1) holds whenever $f$ is continuous at $x$.

THEOREM 1. If $f \in L^{1}$, and $\left\{K_{\alpha}\right\}$ is a smooth approximate identity, then $\left(f * K_{\alpha}\right)(x) \rightarrow f(x)$ a.e. as $\alpha \rightarrow \infty$; specifically, the limit holds for all $x$ for which (1) holds, and so in particular for $x$ where $f$ is continuous.

Proof. Fix $x$, and let $\delta>0$.

$$
\begin{aligned}
f * K_{\alpha}(x) & -f(x)=\int_{-\infty}^{\infty} K_{\alpha}(x-s)(f(s)-f(x)) d s \\
= & \int_{-\infty}^{x-\hat{o}} K_{\alpha}(x-s) f(s) d s-f(x) \int_{-\infty}^{x-\hat{o}} K_{\alpha}(x-s) d s \\
& +\int_{x+\hat{o}}^{\infty} K_{\alpha}(x-s) f(s) d s-f(x) \int_{x+\hat{o}}^{\infty} K_{\alpha}(x-s) d s \\
& +\int_{x-\hat{o}}^{x} K_{\alpha}(x-s)(f(s)-f(x)) d x+\int_{x}^{x+\hat{o}} K_{\alpha}(x-s)(f(s)-f(x)) d s .
\end{aligned}
$$


Let $J_{1}, J_{2}, \cdots, J_{6}$ be the six integrals above, in the order in which they occur.

By Lemma $1, J_{2} \rightarrow 0$ and $J_{4} \rightarrow 0$ as $\alpha \rightarrow \infty$.

$J_{1}$ and $J_{3}$ are similar to each other, and we estimate $J_{1}$ :

$$
\left|J_{1}\right| \leqq\|f\|_{1} \sup \left\{K_{\alpha}(t): t \geqq \delta\right\} \text {. }
$$

Hence $J_{1} \rightarrow 0$ and $J_{3} \rightarrow 0$ as $\alpha \rightarrow \infty$ by Lemma 3 .

Finally we show that $J_{5} \rightarrow 0$ for every $x$ for which (1) holds, and a similar argument holds for $J_{6}$.

Let $x$ be a number for which (1) holds, and let

$$
\beta(s)=\int_{x}^{s}(f(s)-f(x)) d s,
$$

so that $\beta(s) /(s-x) \rightarrow 0$ as $s \rightarrow x$. Then

$$
\begin{aligned}
J_{5} & =\int_{x-\delta}^{x} K_{\alpha}(x-s) d \beta(s) \\
& =K_{\alpha}(0) \beta(x)-K_{\alpha}(\delta) \beta(x-\delta)+\int_{x-\delta}^{x} \beta(s) K_{\alpha}^{\prime}(x-s) d s .
\end{aligned}
$$

Observe that $\beta(x)=0$, that $\beta$ is continuous, and that $K_{\alpha}(\delta) \rightarrow 0$ for any fixed $\delta$, as $\alpha \rightarrow \infty$. Now we estimate the final integral.

$$
\begin{aligned}
\int_{x-\delta}^{x} \beta(s) K_{\alpha}^{\prime}(x-s) d s & \leqq \int_{x-\delta}^{x}\left|\frac{\beta(s)}{s-x}\right|\left|(x-s) K_{\alpha}^{\prime}(x-s)\right| d s \\
& \leqq \max _{x \rightarrow \delta \leqq s \leqq x}\left|\frac{\beta(s)}{s-x}\right| \cdot \int_{0}^{\delta}\left|t K_{\alpha}^{\prime}(t)\right| d t \\
& \leqq B \max _{x \rightarrow \delta \leqq s \leqq x}\left|\frac{\beta(s)}{s-x}\right|,
\end{aligned}
$$

where $B$ is the constant of Lemma 6 . Choose $\delta$ so the right side of (2) is less than $\varepsilon$. Then pick $\alpha_{0}$ so that $\left|K_{\alpha}(\delta) \beta(x-\delta)\right|<\varepsilon$ if $\alpha>\alpha_{0}$. Hence $\left|J_{5}\right|<2 \varepsilon$ if $\alpha>\alpha_{0}$, and we conclude that $J_{5} \rightarrow 0$ as $\alpha \rightarrow \infty$.

In all the applications we take as our net $\left\{K_{\alpha}\right\}$ the translates of some standard approximate identity $\left\{L_{\beta}\right\}$. Hence we state the following simple observation as a lemma.

LEMMA 8. Let $\left\{L_{\beta}\right\}$ be a smooth approximate identity, and let

$$
K_{(\beta, t)}(s)=L_{\beta}(s-t) .
$$

If the indices $(\beta, t)$ are so ordered that $(\beta, t) \rightarrow \infty$ implies $\beta \rightarrow \infty$ and $t \rightarrow 0$, then $\left\{K_{\langle\beta, t\}}\right\}$ satisfies (a), (b), (c), and (d).

For the applications, we so order the pairs $(\beta, t)$ that condition 
(e) also holds. The restrictions imposed by (e) determine the paths along which pointwise convergence occurs.

3. Applications. In this section we apply Theorem 1 to the Poisson kernels for the disc and half-plane, to heat kernels for the half-plane and the first quadrant, and to the Fejér kernel.

(A) The Poisson kernel for the disc.

Let

$$
P_{r}(s)=\frac{1}{2 \pi} \frac{1-r^{2}}{1+r^{2}-2 r \cos s},
$$

where $-\pi<s \leqq \pi$ and $r<1$. The kernels $\left\{P_{r}\right\}$ are a smooth approximate identity as $r \rightarrow 1$ [2, p. 102], [3, p. 32]. Let

$$
K_{\langle r, \theta\rangle}(s)=P_{r}(s-\theta)=\frac{1}{2 \pi} \frac{1-r^{2}}{1+r^{2}-2 r \cos (s-\theta)} .
$$

Here $\alpha=(r, \theta)$ for $0 \leqq r<1$ and $-\pi<\theta \leqq \pi$. The $\alpha$ 's are ordered as follows: fix $A$ and let

$$
(r, \theta)>\left(r^{\prime}, \theta^{\prime}\right) \text { iff } r \geqq r^{\prime},|\theta| \leqq A(1-r),\left|\theta^{\prime}\right| \leqq A\left(1-r^{\prime}\right) .
$$

Clearly $(r, \theta) \rightarrow \infty$ implies $r \rightarrow 1$ and $\theta \rightarrow 0$, so $\left\{K_{(r, \theta)}\right\}$ satisfies (a)-(d). The polar curve $\theta=A(1-r)$ has limiting slope $\pm A$ as $r \rightarrow 1$. Hence $(r, \theta) \rightarrow \infty$ implies that $(r, \theta) \rightarrow(1,0)$ between the lines through $(1,0)$ with slopes $\pm A$. If $F(r, \theta) \rightarrow L$ as $(r, \theta) \rightarrow \infty$, then $\lim _{r \rightarrow 1} F(r, \theta)=L$ and the limit is uniform in $\theta$ for $(r, \theta)$ within the given angle at $(1,0)$. This is formally stronger than the usual statement " $F(r, \theta) \rightarrow L$ along any path to $(1,0)$ within an angle." Actually, the two statements are equivalent, and we will write " $(r, \theta) \rightarrow(1,0)$ in an angle" for $(r, \theta) \rightarrow \infty$.

If $\alpha=(r, \theta)$, then $x_{\alpha}$ of condition (e) is given by $x_{\alpha}=\theta$, and condition (e) is satisfied as follows:

$$
\left|x_{\alpha}\right| K_{\alpha}\left(x_{\alpha}\right)=\frac{|\theta|}{2 \pi} \frac{1-r^{2}}{(1-r)^{2}}=\frac{1+r}{2 \pi} \frac{|\theta|}{1-r} \leqq \frac{A}{\pi} .
$$

THEOREM A. If $f \in L^{1}(-\pi, \pi)$, then

$$
\begin{aligned}
f * K_{\langle r, \theta\rangle}\left(s_{0}\right) & =\frac{1}{2 \pi} \int_{-\pi}^{\pi} \frac{\left(1-r^{2}\right) f(s)}{1+r^{2}-2 r \cos \left(s_{0}-s-\theta\right)} d s \\
& \longrightarrow f\left(s_{0}\right) \text { a.e. as }(r, \theta) \longrightarrow(1,0) \text { in an angle } .
\end{aligned}
$$

To get the usual statement, replace $s_{0}-\theta$ by $\theta$ in the right side of (4) and let 


$$
F(r, \theta)=\frac{1}{2 \pi} \int_{-\pi}^{\pi} \frac{\left(1-r^{2}\right) f(s)}{1+r^{2}-2 r \cos (\theta-s)} d s .
$$

Then $F$ is harmonic in the disc and the classical theorem is:

TheOREM $\mathrm{A}^{\prime}$. If $f \in L^{1}(-\pi, \pi)$, then for almost all $s_{0}, F(r, \theta) \rightarrow$ $f\left(s_{0}\right)$ as $(r, \theta) \rightarrow\left(1, s_{0}\right)$ within an angle at $\left(1, s_{0}\right)$.

To see that condition (e) is an appropriate hypothesis, consider the function $g(z)=\exp (z+1) /(z-1)$, with $g(1)=0$. By [1, Theorem 3.2], (1) holds for this $g$ at $\theta=0$, since $g(r) \rightarrow 0$ as $r \rightarrow 1$. Observe that $|g(z)|=\exp (-P(r, \theta))$, where $z=r e^{i \theta}$. On the circle $r=\cos \theta$, $P(r, \theta)=1 / 2 \pi$, so $|g(z)|$ is a constant different from $g(1)=0$. If $f(\theta)=\operatorname{Re} g\left(e^{i \theta}\right)$ and $F(r, \theta)$ is the Poisson integral of $f$, then $F(r, \theta) \nrightarrow f(0)$ as $(r, \theta) \rightarrow(1,0)$ along the tangential path $r=\cos \theta$, even though (1) holds for $f$ at $\theta=0$.

(B) The Poisson kernel for the half-plane.

Let

$$
P_{y}(s)=\frac{y}{\pi} \frac{1}{y^{2}+s^{2}} .
$$

Here $-\infty<s<\infty$, and $y>0$, and $\left\{P_{y}\right\}$ is an approximate identity as $y \rightarrow 0+[3$, p. 123]. Let

$$
K_{(x, y)}(s)=P_{y}(s-x)=\frac{y}{\pi} \frac{1}{y^{2}+(s-x)^{2}},
$$

where $-\infty<x<\infty$ and $y>0$. Order the pairs $(x, y)$ as follows:

$$
(x, y)>\left(x^{\prime}, y^{\prime}\right) \text { iff } y \leqq y^{\prime}, \quad|x| \leqq A y, \quad{ }^{\prime} x^{\prime} \mid \leqq A y^{\prime} .
$$

Hence $(x, y) \rightarrow \infty$ means $y \rightarrow 0+$ and $(x, y)$ stays between the lines $y= \pm A x$. We will indicate such a limit by " $(x, y) \rightarrow(0,0)$ in an angle."

If $\alpha=(x, y)$, then $x_{\alpha}=x$, and condition (e) becomes

$$
\left|x_{\alpha}\right| K_{\alpha}\left(x_{\alpha}\right)=|x| \cdot \frac{1}{\pi y}=\frac{1}{\pi} \frac{|x|}{y} \leqq \frac{A}{\pi} .
$$

Theorem 1 in this case is

Theorem B. If $f \in L^{1}(-\infty, \infty)$, then

$$
\begin{aligned}
& f * K_{(x, y)}\left(s_{0}\right)=\frac{y}{\pi} \int_{-\infty}^{\infty} \frac{f(s)}{y^{2}+\left(s_{0}-s-x\right)^{2}} d s \\
& \longrightarrow f\left(s_{0}\right) \text { a.e. as }(x, y) \longrightarrow(0,0) \text { in an angle } .
\end{aligned}
$$


Replace $s_{0}-x$ by $x$ in the right side of (6), and let

$$
F(x, y)=\frac{y}{\pi} \int_{-\infty}^{\infty} \frac{f(s)}{y^{2}+(s-x)^{2}} d s .
$$

Then $F$ is harmonic in the upper half-plane, and we have:

THEOREM B'. If $f \in L^{1}(-\infty, \infty)$, then for almost all $s_{0}, F(x, y) \rightarrow$ $f\left(s_{0}\right)$ as $(x, y) \rightarrow\left(s_{0}, 0\right)$ within an angle at $\left(s_{0}, 0\right)$.

(C) Heat kernel for the upper half-plane.

Let

$$
k_{t}(s)=\frac{1}{\sqrt{4 \pi t}} e^{-s^{2} / 4 t},
$$

where $-\infty<s<\infty$, and $t>0$. The family $\left\{k_{t}\right\}$ is an approximate identity as $t \rightarrow 0+[4, \mathrm{p} .31]$, and $k_{t}(s)$ satisfies the heat equation $\partial^{2} u / \partial s^{2}=\partial u / \partial t$. Let

$$
K_{(x, t)}(s)=k_{t}(s-x)=\frac{1}{\sqrt{4 \pi t}} e^{-(s-x)^{2} / 4 t} .
$$

Order the indices $(x, t)$ as follows:

$$
(x, t)>\left(x^{\prime}, t^{\prime}\right) \text { iff } t \leqq t^{\prime},|x| \leqq A \sqrt{t},\left|x^{\prime}\right| \leqq A \sqrt{t^{\prime}} .
$$

Then $(x, t) \rightarrow \infty$ means $t \rightarrow 0+$ and $(x, t)$ lies over the parabola $t=x^{2} / A$. We will write " $(x, t) \rightarrow(0,0)$ over a parabola" for $(x, t) \rightarrow \infty$. If $\alpha=(x, t)$, then $x_{\alpha}=x$, and condition (e) is satisfied as follows:.

$$
\left|x_{\alpha}\right| K_{\alpha}\left(x_{\alpha}\right)=|x| \frac{1}{\sqrt{4 \pi t}} \leqq A / \sqrt{4 \pi} \text {. }
$$

THEOREM C. If $f \in L^{1}(-\infty, \infty)$, then

$$
\begin{aligned}
& f * K_{(x, t)}\left(s_{0}\right)=\frac{1}{\sqrt{4 \pi t}} \int_{-\infty}^{\infty} e^{-\left(s_{0}-s-x\right)^{2} / 4 t} f(s) d s \\
& \longrightarrow f\left(s_{0}\right) \text { a.e. as }(x, t) \rightarrow(0,0) \text { over a parabola } .
\end{aligned}
$$

Replace $s_{0}-x$ by $x$ on the right side of (8), and let

$$
F(x, t)=\frac{1}{\sqrt{4 \pi t}} \int_{-\infty}^{\infty} e^{-(x-s)^{2} / 4 t} f(s) d s .
$$

Then $F(x, t)$ satisfies the heat equation in the upper half-plane, and:

THEOREM $\mathrm{C}^{\prime}$. If $f \in L^{1}(-\infty, \infty)$, then for almost all $s_{0}, F(x, t) \rightarrow$ $f\left(s_{0}\right)$ as $(x, t) \rightarrow\left(s_{0}, 0\right)$ over any parabola $t=A\left(x-s_{0}\right)^{2}$. 
(D) The heat kernel for the first quadrant.

For a function $f$ in $L^{1}(t \geqq 0)$ we want to obtain an extension $f(x, t)$ which satisfies the heat equation in the first quadrant. Since Theorem 1 treats a convolution on $(-\infty, \infty)$ rather than $(0, \infty)$, we first prove a lemma which puts Theorem 1 in accessible form.

LEMMA 9. If $\left\{K_{\alpha}\right\}$ is a smooth approximate identity on $(-\infty$, $\infty)$, and $K_{\alpha} \equiv 0$ on $\left(-\infty, b_{\alpha}\right)$, and $f \in L^{1}(0, N)$ for every $N$, then

$$
\int_{0}^{x-b_{\alpha}} K_{\alpha}(x-s) f(s) d s \longrightarrow f(x) \text { a.e. as } \alpha \longrightarrow \infty \text {. }
$$

Proof. Extend $f$ to the whole line by letting $f \equiv 0$ on $(-\infty, 0)$. Then for $x \geqq b_{\alpha}$,

$$
\begin{aligned}
f * K_{\alpha}(x) & =\int_{-\infty}^{\infty} K_{\alpha}(x-s) f(s) d s \\
& =\int_{0}^{x-b} K_{\alpha}(x-s) f(s) d s .
\end{aligned}
$$

As $\alpha \rightarrow \infty$, $\lim \sup b_{\alpha} \leqq 0$ by Lemma 2. Hence (9) holds for almost all $x$ in $(0, \infty)$.

For $x>0$, the kernel $h_{x}$ is defined as follows:

$$
h_{x}(s)=\left\{\begin{array}{l}
\frac{1}{\sqrt{4 \pi}} \frac{x}{s^{3 / 2}} e^{-x^{2} / 4 s} \text { if } s>0 \\
0 \text { if } s \leqq 0
\end{array}\right.
$$

The function $h_{x}(s)$ satisfies the heat equation $\partial^{2} u / \partial x^{2}=\partial u / \partial s$ everywhere except $(0,0)[4, \mathrm{p} .16]$. For fixed $x>0, h_{x}(s) \equiv 0$ for $s \leqq 0$, and $h_{x}$ has a unique maximum at $s=x^{2} / 6$. The maximum value is

$$
h_{x}\left(x^{2} / 6\right)=\frac{1}{\sqrt{4 \pi}}(6 / e)^{3 / 2} \frac{1}{x^{2}} .
$$

The functions $\left\{h_{x}\right\}$ form an approximate identity as $x \rightarrow 0+[4, p$. 71]. Let

$$
K_{(x, t)}(s)=h_{x}(s-t)=\left\{\begin{array}{l}
\frac{1}{\sqrt{4 \pi}} \frac{x}{(s-t)^{3 / 2}} e^{-x^{2} / 4(s-t)} \text { if } s>t \\
0 \text { if } s \leqq t
\end{array}\right.
$$

Note that $K_{(x, t)} \equiv 0$ on $(-\infty, t)$, so the $b_{\alpha}$ of Lemma 9 is: $b_{(x, t)}=t$. Order the points $(x, t)$ of the right half-plane as follows: fix $B>0$, and let

$$
(x, t) \succ\left(x^{\prime}, t^{\prime}\right) \text { iff } x \leqq x^{\prime},|t| \leqq B x^{2},\left|t^{\prime}\right| \leqq B\left(x^{\prime}\right)^{2}
$$


Hence $(x, t) \rightarrow \infty$ iff $x \rightarrow 0+$ and $(x, t)$ lies in the right half-plane between the parabolas $t= \pm B x^{2}$.

The function $K_{(x, t)}$ has its unique maximum where $s-t=x^{2} / 6$. Hence for $\alpha=(x, t), x_{\alpha}=t+x^{2} / 6$, and the left side of the condition (e) becomes

$$
\left|x_{\alpha}\right| K_{\alpha}\left(x_{\alpha}\right)=\left|t+x^{2} / 6\right| \frac{1}{\sqrt{4 \pi}}\left(\frac{6}{e}\right)^{3 / 2} \frac{1}{x^{2}} .
$$

If $t \geqq-x^{2} / 6$, then (11) becomes, with $c=(6 / e)^{3 / 2} / \sqrt{4 \pi}$,

$$
\left|x_{\alpha}\right| K_{\alpha}\left(x_{\alpha}\right)=c\left(\frac{t}{x^{2}}+\frac{1}{6}\right) \leqq c\left(B+\frac{1}{6}\right) .
$$

If $t<-x^{2} / 6$, then (11) becomes

$$
\left|x_{\alpha}\right| K_{\alpha}\left(x_{\alpha}\right)=c\left(-\frac{t}{x^{2}}-\frac{1}{6}\right) \leqq c B .
$$

Hence (e) holds with constant

$$
A=c\left(B+\frac{1}{6}\right)=\frac{1}{\sqrt{4 \pi}}\left(\frac{6}{e}\right)^{3 / 2}\left(B+\frac{1}{6}\right) .
$$

TheOREM D. If $f \in L^{1}(0, N)$ for every $N$, then for almost all $s_{0}$,

$$
\begin{aligned}
f * K_{(x, t)}\left(s_{0}\right) & =\int_{0}^{s_{0}-t} \frac{1}{\sqrt{4 \pi}} \frac{x}{\left(s_{0}-s-t\right)^{3 / 2}} e^{-x^{2} / 4\left(s_{0}-s-t\right)} f(s) d s \\
& \longrightarrow f\left(s_{0}\right) \text { as }(x, t) \longrightarrow(0,0) \text { between parabolas } .
\end{aligned}
$$

Now replace $s_{0}-t$ by $t$, and let

$$
F(x, t)=\frac{1}{\sqrt{4 \pi}} \int_{0}^{t} \frac{x}{(t-s)^{3 / 2}} e^{-x^{2} / 4(t-s)} f(s) d s .
$$

Then $F(x, t)$ satisfies the heat equation for $x>0, t>0$, and (cf. $[4, \mathrm{p} .78])$ :

TheOREm $\mathrm{D}^{\prime}$. If $f \in L^{1}(0, N)$ for any $N$, then for almost all $s_{0}$, $F(x, t) \rightarrow f\left(s_{0}\right)$ as $(x, t) \rightarrow\left(0, s_{0}\right)$ between any parabolas $t=s_{0} \pm B x^{2}$.

(E) The Fejér kernel.

The Fejér kernel is defined by

$$
L_{n}(s)=\left\{\begin{array}{l}
\frac{1}{n}\left|\frac{\sin \left(\frac{n s}{2}\right)}{\sin \left(\frac{s}{2}\right)}\right|^{2} \text { if } s \neq 0 \\
n \text { if } s=0 .
\end{array}\right.
$$


The family $\left\{L_{n}\right\}$ is smooth approximate identity on $[-\pi, \pi]$ and $f * L_{n}=\sigma_{n}$, the $n$th Cesàro sum of the Fourier series for $f[2$, p. 79], [3, p. 17]. We let $K_{(n, x)}(s)=L_{n}(s-x)$, and order the pairs $(n, x)$ as follows:

$$
(n, x) \succ\left(n^{\prime}, x^{\prime}\right) \quad \text { iff } \quad n \geqq n^{\prime}, \quad n|x| \leqq A, n^{\prime}\left|x^{\prime}\right| \leqq A .
$$

Then $(n, x) \rightarrow \infty$ means $n \rightarrow \infty$ and $|x| \leqq A / n$. The unique maximum of $K_{(n, x)}$ is $K_{(n, x)}(x)=n$, so condition (e) reads

$$
\left|x_{\alpha}\right| K_{\alpha}\left(x_{\alpha}\right)=|x| \cdot n \leqq A \text {. }
$$

Hence $\left\{K_{(n, x)}\right\}$ is a smooth approximate identity and

THEOREM E. If $f \in L^{1}(-\pi, \pi)$, then

$$
\sigma_{n}\left(s_{0}-x\right)=f * K_{(n, x)}\left(s_{0}\right) \longrightarrow f\left(s_{0}\right) \text { a.e. as } n \longrightarrow \infty,
$$

uniformly in $x$ if $|x| \leqq A / n$.

Let $s_{0}$ be a point where the limit above exists, and let $\varepsilon>0$. Then there is $N$ so that $\left|\sigma_{n}\left(s_{0}-x\right)-\sigma_{n}\left(s_{0}\right)\right|<\varepsilon$ if $n \geqq N$ and $|x| \leqq 1 / n$. Hence we have an estimate of the modulus of continuity of $\sigma_{n}$, for large $n$, and this estimate does not depend on $f$, so long as (1) holds at the point in question.

The kind of continuity statement made above for the Cesàro sums can be made for convolutions with any smooth approximate identity. For example, let $f_{r}(\theta)=\left(f * P_{r}\right)(\theta)$ where $P_{r}$ is the Poisson kernel. For $\varepsilon>0$, there is $r_{\varepsilon}$ so that $\left|f_{r}\left(s_{0}\right)-f_{r}\left(s_{0}+\theta\right)\right|<\varepsilon$ for all $r \geqq r_{\varepsilon}$ and all $\theta$ such that $\theta \leqq A(1-r)$. We have an explicit estimate of how the continuity of $f_{r}$ at $s_{0}$ depends on $r$. The estimate is independent of $f$, so long as (1) holds at $s_{0}$.

\section{REFERENCES}

1. T. K. Boehme, M. Rosenfeld, and Max L. Weiss, Relations between bounded analytic functions and their boundary functions, J. London Math. Soc.. (2), 1 (1969), 609-618. 2. R. E. Edwards, Fourier Series, a Modern Introduction, Holt, Rinehart and Winston, Inc., New York, 1967.

3. Kenneth Hoffman, Banach Spaces of Analytic Functions, Prentice-Hall, Inc., Englewood Cliffs, N. J., 1962.

4. D. V. Widder, The Heat Equation, Academic Press, New York, 1975.

Received May 8, 1978.

UNIVERSTTY OF HAWAII

HONOLULU, HI 96822 



\section{PACIFIC JOURNAL OF MATHEMATICS}

EDITORS

DONALD BABBITT (Managing Editor)

University of California

Los Angeles, California 90024

HUGo RossI

University of Utah

Salt Lake City, UT 84112

C. C. MOORE

J. DUGUNDJI

Department of Mathematics

University of Southern California

Los Angeles, California 90007

R. FinN AND J. MiLgraM

Stanford University

Stanford, California 94305

University of California

Berkeley, CA 94720

\section{ASSOCIATE EDITORS}

E. F. BrCKENBACH

B. H. NeUmanN

F. WOLF

K. YoshidA

\section{SUPPORTING INSTITUTIONS}

UNIVERSITY OF BRITISH COLUMBIA

UNIVERSITY OF SOUTHERN CALIFORNIA

CALIFORNIA INSTITUTE OF TECHNOLOGY

STANFORD UNIVERSITY

UNIVERSITY OF CALIFORNIA

UNIVERSITY OF HAWAII

MONTANA STATE UNIVERSITY

UNIVERSITY OF TOKYO

UNIVERSITY OF NEVADA, RENO

UNIVERSITY OF UTAH

NEW MEXICO STATE UNIVERSITY

WASHINGTON STATE UNIVERSITY

OREGON STATE UNIVERSITY

UNIVERSITY OF WASHINGTON

UNIVERSITY OF OREGON 


\section{Pacific Journal of Mathematics}

\section{Vol. 81, No. $1 \quad$ November, 1979}

Thomas E. Armstrong, Simplicial subdivision of infinite-dimensional compact cubes ..................................... 1

Herbert Stanley Bear, Jr., Approximate identities and pointwise convergence ................................

Richard David Bourgin, Partial orderings for integral representations on convex sets with the Radon-Nikodým property..................

Alan Day, Herbert S. Gaskill and Werner Poguntke, Distributive lattices

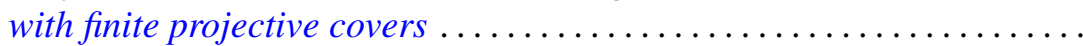

Heneri Amos Murima Dzinotyiweyi and Gerard L. G. Sleijpen, A note on measures on foundation semigroups with weakly compact orbits ......

Ronald James Evans, Resolution of sign ambiguities in Jacobi and Jacobsthal sums ...................................

John Albert Fridy, Tauberian theorems via block dominated matrices ......

Matthew Gould and Helen H. James, Automorphism groups retracting onto

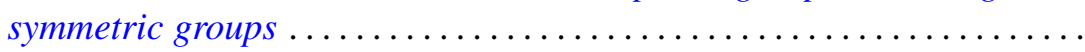

Kurt Kreith, Nonlinear differential equations with monotone solutions . . . . 101

Brian William McEnnis, Shifts on indefinite inner product spaces........ 113

Joseph B. Miles, On entire functions of infinite order with radially

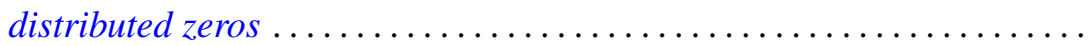

Janet E. Mills, The idempotents of a class of 0-simple inverse semigroups ...............................

Edward Jean Moulis, Jr., Generalizations of the Robertson functions ...

Richard A. Moynihan and Berthold Schweizer, Betweenness relations in probabilistic metric spaces.......................

Stanley Ocken, Perturbing embeddings in codimension two ....

Masilamani Sambandham, On the average number of real zeros of a class of random algebraic curves.

Jerry Searcy and B. Andreas Troesch, A cyclic inequality and a related eigenvalue problem.

Roger R. Smith and Joseph Dinneen Ward, $M$-ideals in $B\left(l_{p}\right)$...

Michel Talagrand, Deux généralisations d'un théorème de I. Namioka ..

Jürgen Voigt, $O n Y$-closed subspaces of $X$, for Banach spaces $X \subset Y$;

existence of alternating elements in subspaces of $C(J)$

Sidney Martin Webster, On mapping an $n$-ball into an $(n+1)$-ball in complex spaces

David J. Winter, Triangulable subalgebras of Lie p-algebras ... 